\title{
Curvature constraints in heterotic Landau-Ginzburg models
}

\section{Richard S. Garavuso}

Physical Sciences, Kingsborough Community College, The City University of New York, 2001 Oriental Boulevard, Brooklyn, NY 11235-2398, U.S.A.

E-mail: richard.garavuso@kbcc.cuny . edu

ABSTRACT: In this paper, we study a class of heterotic Landau-Ginzburg models. We show that the action can be written as a sum of BRST-exact and non-exact terms. The non-exact terms involve the pullback of the complexified Kähler form to the worldsheet and terms arising from the superpotential, which is a Grassmann-odd holomorphic function of the superfields. We then demonstrate that the action is invariant on-shell under supersymmetry transformations up to a total derivative. Finally, we extend the analysis to the case in which the superpotential is not holomorphic. In this case, we find that supersymmetry imposes a constraint which relates the nonholomorphic parameters of the superpotential to the Hermitian curvature. Various special cases of this constraint have previously been used to establish properties of Mathai-Quillen form analogues which arise in the corresponding heterotic Landau-Ginzburg models. There, it was claimed that supersymmetry imposes those constraints. Our goal in this paper is to support that claim. The analysis for the nonholomorphic case also reveals a constraint imposed by supersymmetry that we did not anticipate from studies of Mathai-Quillen form analogues.

KeYwords: Superstrings and Heterotic Strings, Supersymmetry and Duality, Topological Field Theories

ARXIV EPRINT: 1912.01552 


\section{Contents}

1 Introduction 1

2 Action 2

3 Supersymmetry invariance for holomorphic superpotential 3

3.1 BRST-exact and non-exact terms 3

3.2 Supersymmetry invariance of non-exact terms 5

$\begin{array}{llll}4 & \text { Supersymmetry invariance for nonholomorphic superpotential } & 7\end{array}$

\section{Introduction}

A Landau-Ginzburg model is a nonlinear sigma model with a superpotential. For a heterotic Landau-Ginzburg model [1-7], the nonlinear sigma model possesses only $(0,2)$ supersymmetry and the superpotential is a Grassmann-odd function of the superfields which may or may not be holomorphic. These heterotic models have field content consisting of $(0,2)$ bosonic chiral superfields $\Phi^{i}=\left(\phi^{i}, \psi_{+}^{i}\right)$ and $(0,2)$ fermionic chiral superfields $\Lambda^{a}=\left(\lambda_{-}^{a}, H^{a}, E^{a}\right)$, along with their conjugate antichiral superfields $\Phi^{\bar{\imath}}=\left(\phi^{\bar{\imath}}, \psi_{+}^{\bar{\imath}}\right)$ and $\Lambda^{\bar{a}}=\left(\lambda_{-}^{\bar{a}}, \bar{H}^{\bar{a}}, \bar{E}^{\bar{a}}\right)$. The $\phi^{i}$ are local complex coordinates on a Kähler manifold $X$. The $E^{a}$ are local smooth sections of a Hermitian vector bundle $\mathcal{E}$ over $X$, i.e. $E^{a} \in \Gamma(X, \mathcal{E})$. The $H^{a}$ are nonpropagating auxiliary fields. The fermions couple to bundles as follows:

$$
\begin{array}{ll}
\psi_{+}^{i} \in \Gamma\left(K_{\Sigma}^{1 / 2} \otimes \Phi^{*}\left(T^{1,0} X\right)\right), & \lambda_{-}^{a} \in \Gamma\left(\bar{K}_{\Sigma}^{1 / 2} \otimes\left(\Phi^{*} \overline{\mathcal{E}}\right)^{\vee}\right), \\
\psi_{+}^{\bar{\imath}} \in \Gamma\left(K_{\Sigma}^{1 / 2} \otimes\left(\Phi^{*}\left(T^{1,0} X\right)\right)^{\vee}\right), & \lambda_{-}^{\bar{a}} \in \Gamma\left(\bar{K}_{\Sigma}^{1 / 2} \otimes \Phi^{*} \overline{\mathcal{E}}\right),
\end{array}
$$

where $\Phi: \Sigma \rightarrow X$ and $K_{\Sigma}$ is the canonical bundle on the worldsheet $\Sigma$. In [5], heterotic Landau-Ginzburg models with superpotential of the form

$$
W=\Lambda^{a} F_{a},
$$

where $F_{a} \in \Gamma\left(X, \mathcal{E}^{\vee}\right)$, were considered. In this paper, we will study supersymmetry in these heterotic Landau-Ginzburg models with $E^{a}=0$. Such models yield the $(2,2)$ LandauGinzburg models of [8] when $\mathcal{E}=T X$ and $\Lambda^{i} F_{i}=\Lambda^{i} \partial_{i} W^{(2,2)}$, where $W^{(2,2)}$ is the $(2,2)$ superpotential. It was claimed in [7] that, when the superpotential is not holomorphic, supersymmetry imposes a constraint which relates the nonholomorphic parameters of the superpotential to the Hermitian curvature. Our goal in this paper is to support that claim.

This paper is organized as follows: in section 2, we will write down the action for the class of heterotic Landau-Ginzburg models that we are considering. In section 3, for the 
case of a holomorphic superpotential, we will show that the action can be written as a sum of BRST-exact and non-exact terms. We will then demonstrate that the action is invariant on-shell under supersymmetry transformations up to a total derivative. Finally, in section 4, we will extend the analysis to the case in which the superpotential is not holomorphic. In this case, we will show that supersymmetry imposes a constraint which relates the nonholomorphic parameters of the superpotential to the Hermitian curvature. The analysis for this case will also reveal another constraint imposed by supersymmetry.

\section{Action}

Let $X$ be a Kähler manifold with metric $g$, antisymmetric tensor $B$, local real coordinates $\phi^{\mu}$, and local complex coordinates $\phi^{i}$ with complex conjugates $\phi^{\bar{i}}$. Furthermore, let $\mathcal{E}$ be a vector bundle over $X$ with Hermitian fiber metric $h$. We consider the action [5] of a heterotic Landau-Ginzburg model on $X$ with gauge bundle $\mathcal{E}$ :

$$
\begin{aligned}
S=2 t \int_{\Sigma} d^{2} z & {\left[\frac{1}{2}\left(g_{\mu \nu}+i B_{\mu \nu}\right) \partial_{z} \phi^{\mu} \partial_{\bar{z}} \phi^{\nu}+i g_{\overline{\bar{\imath}} i} \psi_{+}^{\bar{\imath}} \bar{D}_{\bar{z}} \psi_{+}^{i}+i h_{a \bar{a}} \lambda_{-}^{a} D_{z} \lambda_{-}^{\bar{a}}\right.} \\
& \left.+F_{i \bar{\imath} a \bar{a}} \psi_{+}^{i} \psi_{+}^{\bar{\imath}} \lambda_{-}^{a} \lambda_{-}^{\bar{a}}+h^{a \bar{a}} F_{a} \bar{F}_{\bar{a}}+\psi_{+}^{i} \lambda_{-}^{a} D_{i} F_{a}+\psi_{+}^{\bar{\imath}} \lambda_{-}^{\bar{a}} \bar{D}_{\bar{\imath}} \bar{F}_{\bar{a}}\right] .
\end{aligned}
$$

Here, $t$ is a coupling constant, $\Sigma$ is a Riemann surface, $F_{a} \in \Gamma\left(X, \mathcal{E}^{\vee}\right)$, and

$$
\begin{aligned}
\bar{D}_{\bar{z}} \psi_{+}^{i} & =\bar{\partial}_{\bar{z}} \psi_{+}^{i}+\bar{\partial}_{\bar{z}} \phi^{j} \Gamma_{j k}^{i} \psi_{+}^{k}, & D_{z} \lambda_{-}^{\bar{a}} & =\partial_{z} \lambda_{-}^{\bar{a}}+\partial_{z} \phi^{\bar{\imath}} A_{\overline{\bar{b}}}^{\bar{a}} \lambda_{-}^{\bar{b}}, \\
D_{i} F_{a} & =\partial_{i} F_{a}-A_{i a}^{b} F_{b}, & \bar{D}_{\bar{\imath}} \bar{F}_{\bar{a}} & =\bar{\partial}_{\bar{\imath}} \bar{F}_{\bar{a}}-A_{\bar{\imath} \bar{a}}^{\bar{b}} \bar{F}_{\bar{b}}, \\
A_{i a}^{b} & =h^{b \bar{b}} h_{\bar{b} a, i}, & A_{\bar{\imath} \bar{a}}^{\bar{b}} & =h^{\bar{b} b} h_{b \bar{a}, \bar{l}}, \\
\Gamma_{j k}^{i} & =g^{i \bar{\imath}} g_{\bar{\imath} k, j}, & F_{i \bar{\imath} a \bar{a}} & =h_{a \bar{b}} A_{\bar{\imath} \bar{a}, i}^{\bar{b}} .
\end{aligned}
$$

The fermions couple to bundles in the manner described in section 1.

The action (2.1) is invariant on-shell under the supersymmetry transformations

$$
\begin{aligned}
\delta \phi^{i} & =i \alpha_{-} \psi_{+}^{i}, \\
\delta \phi^{\bar{\imath}} & =i \tilde{\alpha}_{-} \psi_{+}^{\bar{\imath}} \\
\delta \psi_{+}^{i} & =-\tilde{\alpha}_{-} \bar{\partial}_{\bar{z}} \phi^{i} \\
\delta \psi_{+}^{\bar{\imath}} & =-\alpha_{-} \partial_{z} \phi^{\bar{\imath}} \\
\delta \lambda_{-}^{a} & =-i \alpha_{-} \psi_{+}^{j} A_{j b}^{a} \lambda_{-}^{b}+i \alpha_{-} h^{a \bar{a}} \bar{F}_{\bar{a}} \\
\delta \lambda_{-}^{\bar{a}} & =-i \tilde{\alpha}_{-} \psi_{+}^{\bar{j}} A_{\bar{\jmath} \bar{b}}^{\bar{a}} \lambda_{-}^{\bar{b}}+i \tilde{\alpha}_{-} h^{\bar{a} a} F_{a}
\end{aligned}
$$

up to a total derivative. In section 3, we will demonstrate this invariance for the case in which the superpotential is holomorphic. In section 4, we will extend the analysis to the case in which the superpotential is not holomorphic. There, we will find that supersymmetry imposes a constraint which relates the nonholomorphic parameters of the superpotential to the Hermitian curvature. We will also find another constraint imposed by supersymmetry. 


\section{Supersymmetry invariance for holomorphic superpotential}

In this section, we will show that, when the superpotential is holomorphic, the action (2.1) is invariant on-shell under the supersymmetry transformations (2.2) up to a total derivative. For these purposes, it is sufficient to set $\tilde{\alpha}_{-}=0,{ }^{1}$ yielding

$$
\begin{aligned}
\delta \phi^{i} & =i \alpha_{-} \psi_{+}^{i}, & \delta \phi^{\bar{\imath}} & =0, \\
\delta \psi_{+}^{i} & =0, & \delta \psi_{+}^{\bar{\imath}} & =-\alpha_{-} \partial_{z} \phi^{\bar{\imath}}, \\
\delta \lambda_{-}^{a} & =-i \alpha_{-} \psi_{+}^{j} A_{j b}^{a} \lambda_{-}^{b}+i \alpha_{-} h^{a \bar{a}} \bar{F}_{\bar{a}}, & \delta \lambda_{-}^{\bar{a}} & =0 .
\end{aligned}
$$

With this simplification, using the $\lambda_{-}^{a}$ equation of motion, ${ }^{2}$ we will show in section 3.1 that the action (2.1) can be written

$$
S=i t \int_{\Sigma} d^{2} z\{Q, V\}+t \int_{\Sigma} \Phi^{*}(K)+2 t \int_{\Sigma} d^{2} z\left(\psi_{+}^{\bar{\imath}} \lambda_{-}^{\bar{a}} \bar{D}_{\bar{\imath}} \bar{F} \bar{a}-\psi_{+}^{i} \lambda_{-}^{a} D_{a} F_{a}\right)
$$

where $Q$ is the BRST operator, $d^{2} z=-i d z \wedge d \bar{z}$,

$$
V=2\left(g_{\bar{\imath} i} \psi_{+}^{\bar{\imath}} \bar{\partial} \bar{z} \phi^{i}+i \lambda_{-}^{a} F_{a}\right)
$$

and

$$
\int_{\Sigma} \Phi^{*}(K)=\int_{\Sigma} d^{2} z\left(g_{i \bar{\imath}}+i B_{i \bar{\imath}}\right)\left(\partial_{z} \phi^{i} \bar{\partial}_{\bar{z}} \phi^{\bar{\imath}}-\bar{\partial}_{\bar{z}} \phi^{i} \partial_{z} \phi^{\bar{\imath}}\right)
$$

is the integral over the worldsheet $\Sigma$ of the pullback to $\Sigma$ of the complexified Kähler form $K=-i\left(g_{i \bar{\imath}}+i B_{i \bar{\imath}}\right) d \phi^{i} \wedge d \phi^{\bar{\imath}}$. Since $\delta f=-i \alpha_{-}\{Q, f\}$, where $f$ is any field, the $Q$-exact part of (3.2) is $\delta$-exact and hence $\delta$-closed. In section 3.2, we will complete our argument by establishing that the remaining terms are $\delta$-closed on shell up to a total derivative.

\subsection{BRST-exact and non-exact terms}

Let us now derive (3.2). The BRST transformations are

$$
\begin{aligned}
\left\{Q, \phi^{i}\right\} & =-\psi_{+}^{i}, & & \left\{Q, \phi^{\bar{\imath}}\right\}=0, \\
\left\{Q, \psi_{+}^{i}\right\} & =0, & & \left\{Q, \psi_{+}^{\bar{\imath}}\right\}=-i \partial_{z} \phi^{\bar{\imath}}, \\
\left\{Q, \lambda_{-}^{a}\right\} & =\psi_{+}^{j} A_{j b}^{a} \lambda_{-}^{b}-h^{a \bar{a}} \bar{F}_{\bar{a}}, & & \left\{Q, \lambda_{-}^{\bar{a}}\right\}=0 .
\end{aligned}
$$

\footnotetext{
${ }^{1}$ The calculations for the case in which $\alpha_{-}=0$ and $\tilde{\alpha}_{-} \neq 0$ are analogous to those we will perform explicitly for the case in which $\alpha_{-} \neq 0$ and $\tilde{\alpha}_{-}=0$. The general case, i.e. $\alpha_{-}$and $\tilde{\alpha}_{-}$both nonzero, is obtained by combining the above two cases.

${ }^{2}$ This is valid because we have integrated out the auxiliary fields $H^{a}$.
} 
Now, we compute

$$
\begin{aligned}
\frac{\{Q, V\}}{2}= & \left\{Q, g_{\bar{\imath} i} \psi_{+}^{\bar{\imath}} \bar{\partial}_{\bar{z}} \phi^{i}+i \lambda_{-}^{a} F_{a}\right\} \\
= & \left\{Q, g_{\bar{\imath} i}\right\} \psi_{+}^{\bar{\imath}} \bar{\partial}_{\bar{z}} \phi^{i}+g_{\bar{\imath} i}\left\{Q, \psi_{+}^{\bar{\imath}}\right\} \bar{\partial}_{\bar{z}} \phi^{i}-g_{\bar{\imath} i} \psi_{+}^{\bar{\imath}} \bar{\partial}_{\bar{z}}\left\{Q, \phi^{i}\right\} \\
& +i\left\{Q, \lambda_{-}^{a}\right\} F_{a}-i \lambda_{-}^{a}\left\{Q, F_{a}\right\} \\
= & \left(g_{\bar{\imath} i, k}\left\{Q, \phi^{k}\right\}\right) \psi_{+}^{\bar{\imath}} \bar{\partial}_{\bar{z}} \phi^{i}+g_{\bar{\imath} i}\left(-i \partial_{z} \phi^{\bar{\imath}}\right) \bar{\partial}_{\bar{z}} \phi^{i}-g_{\bar{\imath} i} \psi_{+}^{\bar{\imath}} \bar{\partial}_{\bar{z}}\left(-\psi_{+}^{i}\right) \\
& +i\left(\psi_{+}^{j} A_{j b}^{a} \lambda_{-}^{b}-h^{a \bar{a}} \bar{F}_{\bar{a}}\right) F_{a}-i \lambda_{-}^{a}\left(F_{a, k}\left\{Q, \phi^{k}\right\}\right) \\
= & g_{\bar{\imath} j} \Gamma_{i k}^{j}\left(-\psi_{+}^{k}\right) \psi_{+}^{\bar{\imath}} \bar{\partial}_{\bar{z}} \phi^{i}-i g_{\bar{\imath} i} \partial_{z} \phi^{\bar{\imath}} \bar{\partial}_{\bar{z}} \phi^{i}+g_{\bar{\imath} i} \psi_{+}^{\bar{\imath}} \bar{\partial}_{\bar{z}} \psi_{+}^{i} \\
& +i \psi_{+}^{j} A_{j b}^{a} \lambda_{-}^{b} F_{a}-i h^{a \bar{a}} \bar{F}_{\bar{a}} F_{a}-i \lambda_{-}^{a} F_{a, k}\left(-\psi_{+}^{k}\right) \\
= & -i g_{\bar{\imath} i} \partial_{z} \phi^{\bar{\imath}} \bar{\partial}_{\bar{z}} \phi^{i}+g_{\bar{\imath} i} \psi_{+}^{\bar{\imath}}\left(\bar{\partial}_{\bar{z}} \psi_{+}^{i}+\bar{\partial}_{\bar{z}} \phi^{j} \Gamma_{j k}^{i} \psi_{+}^{k}\right) \\
& -i h^{a \bar{a}} \bar{F}_{\bar{a}} F_{a}-i \psi_{+}^{i} \lambda_{-}^{a}\left(\partial_{i} F_{a}-A_{i a}^{b} F_{b}\right) \\
= & -i g_{\bar{\imath} i} \partial_{z} \phi^{\bar{\imath}} \bar{\partial}_{\bar{z}} \phi^{i}+g_{\bar{\imath} i} \psi_{+}^{\bar{\imath}} \bar{D}_{\bar{z}} \psi_{+}^{i}-i h^{a \bar{a}} \bar{F}_{\bar{a}} F_{a}-i \psi_{+}^{i} \lambda_{-}^{a} D_{i} F_{a},
\end{aligned}
$$

where we have used $g_{\bar{\imath} i, k}=g_{\bar{i} j} \Gamma_{i k}^{j}$ in the fourth step. It follows that

$$
i t \int_{\Sigma} d^{2} z\{Q, V\}=2 t \int_{\Sigma} d^{2} z\left(g_{\bar{\imath} i} \partial_{z} \phi^{\bar{\imath}} \bar{\partial} \bar{z} \phi^{i}+i g_{\bar{\imath} i} \psi_{+}^{\bar{\imath}} \overline{D_{\bar{z}}} \psi_{+}^{i}+h^{a \bar{a}} \overline{F_{\bar{a}}} F_{a}+\psi_{+}^{i} \lambda_{-}^{a} D_{i} F_{a}\right) .
$$

Using the identity

$$
\int_{\Sigma} d^{2} z g_{\bar{\imath} i} \partial_{z} \phi^{\bar{\imath}} \bar{\partial}_{\bar{z}} \phi^{i}=\int_{\Sigma} d^{2} z \frac{1}{2}\left(g_{\mu \nu}+i B_{\mu \nu}\right) \partial_{z} \phi^{\mu} \partial_{\bar{z}} \phi^{\nu}-\frac{1}{2} \int_{\Sigma} \Phi^{*}(K),
$$

we obtain

$$
\begin{aligned}
i t \int_{\Sigma} d^{2} z\{Q, V\} & \\
= & 2 t \int_{\Sigma} d^{2} z\left[\frac{1}{2}\left(g_{\mu \nu}+i B_{\mu \nu}\right) \partial_{z} \phi^{\mu} \bar{\partial}_{\bar{z}} \phi^{\nu}+i g_{\bar{\imath} i} \psi_{+}^{\bar{\imath}} \bar{D}_{\bar{z}} \psi_{+}^{i}+h^{a \bar{a}} \bar{F}_{\bar{a}} F_{a}+\psi_{+}^{i} \lambda_{-}^{a} D_{i} F_{a}\right] \\
& -t \int_{\Sigma} \Phi^{*}(K) \\
= & S-t \int_{\Sigma} \Phi^{*}(K)-2 t \int_{\Sigma} d^{2} z\left(i h_{\bar{a} a} \lambda_{-}^{a} D_{z} \lambda_{-}^{\bar{a}}+F_{i \bar{\imath} a \bar{a}} \psi_{+}^{i} \psi_{+}^{\bar{\imath}} \lambda_{-}^{a} \lambda_{-}^{\bar{a}}+\psi_{+}^{\bar{\imath}} \lambda_{-}^{\bar{a}} \bar{D}_{\bar{\imath}} \bar{F}_{\bar{a}}\right),
\end{aligned}
$$

and hence

$$
\begin{aligned}
S= & i t \int_{\Sigma} d^{2} z\{Q, V\}+t \int_{\Sigma} \Phi^{*}(K) \\
& +2 t \int_{\Sigma} d^{2} z\left(i h_{\bar{a} a} \lambda_{-}^{a} D_{z} \lambda_{-}^{\bar{a}}+F_{i \bar{\imath} a \bar{a}} \psi_{+}^{i} \psi_{+}^{\bar{\imath}} \lambda_{-}^{a} \lambda_{-}^{\bar{a}}+\psi_{+}^{\overline{\overline{ }}} \lambda_{-}^{\bar{a}} D_{\bar{\imath}} \bar{F}_{\bar{a}}\right) .
\end{aligned}
$$

An analogous result was found in [4] for the case in which the gauge fields are absent and $B=0$. Finally, using the $\lambda_{-}^{a}$ equation of motion

$$
\lambda_{-}^{a}: \quad i h_{a \bar{a}} D_{z} \lambda_{-}^{\bar{a}}+F_{i \bar{a} a \bar{a}} \psi_{+}^{i} \psi_{+}^{\bar{\imath}} \lambda_{-}^{\bar{a}}-\psi_{+}^{i} D_{i} F_{a}=0,
$$


we obtain

$$
i h_{a \bar{a}} \lambda_{-}^{a} D_{z} \lambda_{-}^{\bar{a}}+F_{i \bar{\imath} a \bar{a}} \psi_{+}^{i} \psi_{+}^{\bar{\imath}} \lambda_{-}^{a} \lambda_{-}^{\bar{a}}=-\psi_{+}^{i} \lambda_{-}^{a} D_{i} F_{a}
$$

and hence

$$
S=i t \int_{\Sigma} d^{2} z\{Q, V\}+t \int_{\Sigma} \Phi^{*}(K)+2 t \int_{\Sigma} d^{2} z\left(\psi_{+}^{\bar{\imath}} \lambda_{-}^{\bar{a}} \bar{D}_{\bar{\imath}} \bar{F}_{\bar{a}}-\psi_{+}^{i} \lambda_{-}^{a} D_{i} F_{a}\right)
$$

which is (3.2).

\subsection{Supersymmetry invariance of non-exact terms}

Let us now complete our argument that the action (3.2) is $\delta$-closed on shell up to a total derivative. As we previously noted, the $Q$-exact part of (3.2) is $\delta$-exact and hence $\delta$-closed. For the non-exact term of (3.2) involving $\Phi^{*}(K)$, note that

$$
\int_{\Sigma} \Phi^{*}(K)=\int_{\Phi(\Sigma)} K=\int_{\Phi(\Sigma)}\left[-i\left(g_{i \bar{\imath}}+i B_{i \bar{\imath}}\right)\right] d \phi^{i} \wedge d \phi^{\bar{\imath}}
$$

and $K$ satisfies

$$
\partial K=-i \partial_{k}\left(g_{i \bar{\imath}}+i B_{i \bar{\imath}}\right) d \phi^{k} \wedge d \phi^{i} \wedge d \phi^{\bar{\imath}}=0 .
$$

Thus,

$$
\delta\left[\Phi^{*}(K)\right]=\left[\Phi^{*}(K)\right]_{k} \delta \phi^{k}=0 .
$$

It remains to consider the non-exact expression of (3.2) involving $\psi_{+}^{\bar{\imath}} \lambda_{-}^{\bar{a}} \bar{D}_{\bar{\imath}} \bar{F}_{\bar{a}}-\psi_{+}^{i} \lambda_{-}^{a} D_{i} F_{a}$. First, we compute

$$
\begin{aligned}
& \delta\left(\psi_{+}^{\bar{\imath}} \lambda_{-}^{\bar{a}} \bar{D}_{\bar{\imath}} \bar{F}_{\bar{a}}\right)=\left(\delta \psi_{+}^{\bar{\imath}}\right) \lambda_{-}^{\bar{a}} \bar{D}_{\bar{\imath}} \bar{F}_{\bar{a}}+\psi_{+}^{\bar{\imath}}\left(\delta \lambda_{-}^{\bar{a}}\right) \bar{D}_{\bar{\imath}} \bar{F}_{\bar{a}}+\psi_{+}^{\bar{\imath}} \lambda_{i}^{\bar{a}}\left[\delta\left(\bar{D}_{\bar{\imath}} \bar{F}_{\bar{a}}\right)\right] \\
& =\left(-\alpha_{-} \partial_{z} \phi^{\bar{\imath}}\right) \lambda_{-}^{\bar{a}} \bar{D}_{\bar{\imath}} \bar{F}_{\bar{a}}+\psi_{+}^{\bar{\imath}} \lambda_{-}^{\bar{a}}\left[\delta\left(\bar{\partial}_{\bar{\imath}} \bar{F}_{\bar{a}}-A_{\bar{\imath} \bar{a}}^{\bar{b}} \bar{F}_{\bar{b}}\right)\right] \\
& =\left(-\alpha_{-} \partial_{z} \phi^{\bar{\imath}}\right) \lambda_{-}^{\bar{a}} \bar{D}_{\bar{\imath}} \bar{F}_{\bar{a}}+\psi_{+}^{\bar{\imath}} \lambda_{-}^{\bar{a}}\left[\bar{\partial}_{\bar{\imath}}\left(\delta \bar{F}_{\bar{a}}\right)-\left(\delta A_{\bar{\imath} \bar{a}}^{\bar{b}}\right) \bar{F}_{\bar{b}}-A_{\bar{\imath} \bar{a}}^{\bar{b}}\left(\delta \bar{F}_{\bar{b}}\right)\right] \\
& =\left(-\alpha_{-} \partial_{z} \phi^{\bar{\imath}}\right) \lambda_{-}^{\bar{a}} \bar{D}_{\bar{\imath}} \bar{F}_{\bar{a}} \\
& +\psi_{+}^{\bar{\imath}} \lambda_{-}^{\bar{a}}\left\{\bar{\partial}_{\bar{\imath}}\left[\bar{F}_{\bar{a}, k}\left(\delta \phi^{k}\right)\right]-\left[A_{\bar{\imath} \bar{a}, k}^{\bar{b}}\left(\delta \phi^{k}\right)\right] \bar{F}_{\bar{b}}-A_{\bar{\imath} \bar{a}}^{\bar{b}}\left[\bar{F}_{\bar{b}, k}\left(\delta \phi^{k}\right)\right]\right\} \\
& =\left(-\alpha_{-} \partial_{z} \phi^{\bar{\imath}}\right) \lambda_{-}^{\bar{a}} \bar{D}_{\bar{\imath}} \bar{F}_{\bar{a}}-\psi_{+}^{\bar{\imath}} \lambda_{-}^{\bar{a}} A_{\bar{\imath} \bar{a}, k}^{\bar{b}}\left(i \alpha_{-} \psi_{+}^{k}\right) \bar{F}_{\bar{b}},
\end{aligned}
$$


where we have used $\bar{F}_{\bar{a}, k}=0$ in the last step. Now, we compute

$$
\begin{aligned}
& \delta\left(-\psi_{+}^{i} \lambda_{-}^{a} D_{i} F_{a}\right)=-\left(\delta \psi_{+}^{i}\right) \lambda_{-}^{a} D_{i} F_{a}-\psi_{+}^{i}\left(\delta \lambda_{-}^{a}\right) D_{i} F_{a}-\psi_{+}^{i} \lambda_{-}^{a}\left[\delta\left(D_{i} F_{a}\right)\right] \\
& =-\psi_{+}^{i}\left(-i \alpha_{-} \psi_{+}^{j} A_{j b}^{a} \lambda_{-}^{b}+i \alpha_{-} h^{a \bar{a}} \bar{F}_{\bar{a}}\right) D_{i} F_{a}-\psi_{+}^{i} \lambda_{-}^{a}\left[\delta\left(\partial_{i} F_{a}-A_{i a}^{b} F_{b}\right)\right] \\
& =-\psi_{+}^{i}\left(-i \alpha_{-} \psi_{+}^{j} A_{j b}^{a} \lambda_{-}^{b}\right)\left(\partial_{i} F_{a}-A_{i a}^{c} F_{c}\right) \\
& -\psi_{+}^{i}\left(i \alpha_{-} h^{a \bar{a}} \bar{F}_{\bar{a}}\right) D_{i} F_{a}-\psi_{+}^{a} \lambda_{-}^{a}\left[\partial_{i}\left(\delta F_{a}\right)-\left(\delta A_{i a}^{b}\right) F_{b}-A_{i a}^{b}\left(\delta F_{b}\right)\right] \\
& =-\psi_{+}^{i}\left(-i \alpha_{-} \psi_{+}^{j} A_{j b}^{a} \lambda_{-}^{b}\right) \partial_{i} F_{a} \\
& -\psi_{+}^{i}\left[-i \alpha_{-} \psi_{+}^{j}\left(h^{a \bar{b}} \partial_{j} h_{\bar{b} b}\right) \lambda_{-}^{b}\right]\left[-\left(h^{c \bar{c}} \partial_{i} h_{\bar{c} a}\right) F_{c}\right] \\
& +i \alpha_{-} h^{a \bar{b}} \bar{F}_{\bar{b}}\left(\psi_{+}^{i} D_{i} F_{a}\right) \\
& -\psi_{+}^{i} \lambda_{-}^{a}\left\{\partial_{i}\left[F_{a, k}\left(\delta \phi^{k}\right)\right]-\left[A_{i a, k}^{b}\left(\delta \phi^{k}\right)\right] F_{b}-A_{i a}^{b}\left[F_{b, k}\left(\delta \phi^{k}\right)\right]\right\} \\
& =-\psi_{+}^{i}\left(-i \alpha_{-} \psi_{+}^{j} A_{j b}^{a} \lambda_{-}^{b}\right) \partial_{i} F_{a} \\
& -\psi_{+}^{i}\left[-i \alpha_{-} \psi_{+}^{j}\left(h^{a \bar{b}} \partial_{j} h_{\bar{b} b}\right) \lambda_{-}^{b}\right]\left[-\left(-h_{\bar{c} a} \partial_{i} h^{c \bar{c}}\right) F_{c}\right] \\
& +i \alpha_{-} h^{a \bar{b}} \bar{F}_{\bar{b}}\left(i h_{a \bar{a}} D_{z} \lambda_{-}^{\bar{a}}+F_{i \bar{\imath} a \bar{a}} \psi_{+}^{i} \psi_{+}^{\bar{\imath}} \lambda_{-}^{\bar{a}}\right) \\
& -\psi_{+}^{i} \lambda_{-}^{a}\left[\partial_{i} F_{a, k}\left(i \alpha_{-} \psi_{+}^{k}\right)-A_{i a, k}^{b}\left(i \alpha_{-} \psi_{+}^{k}\right) F_{b}-A_{i a}^{b} F_{b, k}\left(i \alpha_{-} \psi_{+}^{k}\right)\right] \\
& =-\psi_{+}^{i}\left(-i \alpha_{-} \psi_{+}^{j} A_{j b}^{a} \lambda_{-}^{b}\right) \partial_{i} F_{a} \\
& -\psi_{+}^{i}\left[-i \alpha_{-} \psi_{+}^{j}\left(\partial_{i} h^{a \bar{b}} \partial_{j} h_{\bar{b} b}\right) \lambda_{-}^{b} F_{a}\right] \\
& -\alpha_{-} \bar{F}_{\bar{a}} D_{z} \lambda_{-}^{\bar{a}}+\left(i \alpha_{-} h^{a \bar{b}} \bar{F}_{\bar{b}}\right) F_{i \bar{\imath} a \bar{a}} \psi_{+}^{i} \psi_{+}^{\bar{\imath}} \lambda_{-}^{\bar{a}} \\
& +\psi_{+}^{i}\left(-i \alpha_{-} \psi_{+}^{j} A_{j b, i}^{a} \lambda_{-}^{b} F_{a}\right)+\psi_{+}^{i}\left(-i \alpha_{-} \psi_{+}^{j} A_{j b}^{a} \lambda_{-}^{b}\right) \partial_{i} F_{a} \\
& =-\psi_{+}^{i}\left[-i \alpha_{-} \psi_{+}^{j}\left(\partial_{i} h^{a \bar{b}} \partial_{j} h_{\bar{b} b}\right) \lambda_{-}^{b} F_{a}\right] \\
& -\alpha_{-} \overline{F_{\bar{a}}} D_{z} \lambda_{-}^{\bar{a}}+\left(i \alpha_{-} h^{a \bar{b}} \bar{F}_{\bar{b}}\right) F_{i \bar{\imath} a \bar{a}} \psi_{+}^{i} \psi_{+}^{\bar{\imath}} \lambda_{-}^{\bar{a}} \\
& +\psi_{+}^{i}\left[-i \alpha_{-} \psi_{+}^{j}\left(\partial_{i} h^{a \bar{b}} \partial_{j} h_{\bar{b} b}+h^{a \bar{b}} \partial_{i} \partial_{j} h_{\bar{b} b}\right) \lambda_{-}^{b} F_{a}\right] \\
& =-\alpha_{-} \overline{F_{\bar{a}}} D_{z} \lambda_{-}^{\bar{a}}+\left(i \alpha_{-} h^{a \bar{b}} \bar{F}_{\bar{b}}\right) F_{i \bar{\imath} a \bar{a}} \psi_{+}^{i} \psi_{+}^{\bar{\imath}} \lambda_{-}^{\bar{a}} \text {. }
\end{aligned}
$$

where we have used $A_{j b}^{a}=h^{a \bar{b}} \partial_{j} h_{\bar{b} b}$ in the fourth step, the identity $h^{c \bar{c}} \partial_{i} h_{\bar{c} a}=-h_{\bar{c} a} \partial_{i} h^{c \bar{c}}$ and the $\lambda_{-}^{a}$ equation of motion (3.7) in the fifth step, $\psi_{+}^{i} \lambda_{-}^{a} \partial_{i} F_{a, k}\left(i \alpha_{-} \psi_{+}^{k}\right)=0$ in the sixth step, $A_{j b, i}^{a}=\partial_{i} h^{a \bar{b}} \partial_{j} h_{\bar{b} b}+h^{a \bar{b}} \partial_{i} \partial_{j} h_{\bar{b} b}$ in the seventh step, and

$$
\psi_{+}^{i}\left[i \alpha_{-} \psi_{+}^{j}\left(h^{a \bar{b}} \partial_{i} \partial_{j} h_{\bar{b} b}\right) \lambda_{-}^{b} F_{a}\right]=0
$$


in the last step. Note that the first term on the right-hand side of (3.10) cancels the first term on the right-hand side of (3.9) up to a total derivative:

$$
\begin{aligned}
-\alpha_{-} \bar{F}_{\bar{a}} D_{z} \lambda_{-}^{\bar{a}} & =-\alpha_{-} \bar{F}_{\bar{a}}\left(\partial_{z} \lambda_{-}^{\bar{a}}+\partial_{z} \phi^{\bar{\imath}} A_{\bar{\imath} \bar{b}}^{\bar{a}} \lambda_{-}^{\bar{b}}\right) \\
& =\alpha_{-}\left(\bar{F}_{\bar{a}, k} \partial_{z} \phi^{k}+\bar{F}_{\bar{a}, \bar{k}} \partial_{z} \phi^{\bar{k}}\right) \lambda_{-}^{\bar{a}}-\alpha_{-} \partial_{z}\left(\bar{F}_{\bar{a}} \lambda_{-}^{\bar{a}}\right)-\alpha_{-} \bar{F}_{\bar{a}} \partial_{z} \phi^{\bar{\imath}} A_{\bar{\imath} \bar{b}}^{\bar{a}} \lambda_{-}^{\bar{b}} \\
& =\left(\alpha_{-} \partial_{z} \phi^{\bar{\imath}}\right) \lambda_{-}^{\bar{a}}\left(\bar{\partial}_{\bar{\imath}} \bar{F}_{\bar{a}}-A_{\bar{\imath} \bar{a}}^{\bar{b}} \bar{F}_{\bar{b}}\right)-\alpha_{-} \partial_{z}\left(\bar{F}_{\bar{a}} \lambda_{-}^{\bar{a}}\right) \\
& =\left(\alpha_{-} \partial_{z} \phi^{\bar{\imath}}\right) \lambda_{-}^{\bar{a}} \bar{D}_{\bar{\imath}} \bar{F}_{\bar{a}}-\alpha_{-} \partial_{z}\left(\bar{F}_{\bar{a}} \lambda_{-}^{\bar{a}}\right)
\end{aligned}
$$

where we used $\bar{F}_{\bar{a}, k}=0$ in the fourth step. Furthermore, the second term on the right-hand side of (3.10) cancels the second term on the right-hand side of (3.9):

$$
\begin{aligned}
\left(i \alpha_{-} h^{a \bar{b}} \bar{F}_{\bar{b}}\right) F_{\bar{\imath} \bar{\imath} a \bar{a}} \psi_{+}^{i} \psi_{+}^{\bar{i}} \lambda_{-}^{\bar{a}} & =\left(i \alpha_{-} h^{a \bar{c}} \bar{F}_{\bar{c}}\right)\left(h_{a \bar{b}} A_{\bar{\imath}}^{\bar{b}}, i\right) \psi_{+}^{\bar{\imath}} \lambda_{-}^{\bar{a}} \psi_{+}^{i} \\
& =\psi_{+}^{\bar{\imath}} \lambda_{-}^{\bar{a}} A_{\bar{\imath} \bar{a}, k}^{\bar{b}}\left(i \alpha_{-} \psi_{+}^{k}\right) \bar{F}_{\bar{b}},
\end{aligned}
$$

where we have used $F_{i \bar{\imath} a \bar{a}}=h_{a \bar{b}} A_{\bar{\imath} \bar{a}, i}$ in the first step. It follows that (3.10) cancels (3.9) up to a total derivative, i.e.

$$
\delta\left(-\psi_{+}^{i} \lambda_{-}^{a} D_{i} F_{a}\right)=-\delta\left(\psi_{+}^{\bar{\imath}} \lambda_{-}^{\bar{a}} \bar{D}_{\bar{\imath}} \bar{F}_{\bar{a}}\right)-\alpha_{-} \partial_{z}\left(\bar{F}_{\bar{a}} \lambda_{-}^{\bar{a}}\right) .
$$

This completes our argument.

\section{Supersymmetry invariance for nonholomorphic superpotential}

In this section, we will extend the analysis of section 3 to the case in which the superpotential is not holomorphic. This requires revisting the steps in (3.9) and (3.11) where we used $\bar{F}_{\bar{a}, k}=0$. Allowing for $\bar{F}_{\bar{a}, k} \neq 0,(3.11)$ becomes

$$
-\alpha_{-} \bar{F}_{\bar{a}} D_{z} \lambda_{-}^{\bar{a}}=\left(\alpha_{-} \partial_{z} \phi^{\bar{\imath}}\right) \lambda_{-}^{\bar{a}} \bar{D}_{\bar{\imath}} \bar{F}_{\bar{a}}-\alpha_{-} \partial_{z}\left(\bar{F}_{\bar{a}} \lambda_{-}^{\bar{a}}\right)+\alpha_{-} \bar{F}_{\bar{a}, k} \partial_{z} \phi^{k} \lambda_{-}^{\bar{a}} .
$$

It follows that the cancellation described by (3.11) will still apply provided that the constraint

$$
\bar{F}_{\bar{a}, k} \partial_{z} \phi^{k} \lambda_{-}^{\bar{a}}=0
$$

is satisfied. Furthermore, in the next to last line of (3.9), we now have

$$
\begin{aligned}
& \psi_{+}^{\bar{\imath}} \lambda_{-}^{\bar{a}}\left\{\bar{\partial}_{\bar{\imath}}\left[\bar{F}_{\bar{a}, k}\left(\delta \phi^{k}\right)\right]-A_{\bar{\imath} \bar{a}}^{\bar{b}}\left[\bar{F}_{\bar{b}, k}\left(\delta \phi^{k}\right)\right]\right\} \\
& =\psi_{+}^{\bar{\imath}} \lambda_{-}^{\bar{a}}\left\{\bar{\partial}_{\bar{\imath}}\left[\bar{F}_{\bar{a}, k}\left(i \alpha_{-} \psi_{+}^{k}\right)\right]-A_{\bar{\imath} \bar{a}}^{\bar{b}} \bar{F}_{\bar{b}, k}\left(i \alpha_{-} \psi_{+}^{k}\right)\right\} \\
& =\psi_{+}^{\bar{\imath}} \lambda_{-}^{\bar{a}}\left(\bar{\partial}_{\bar{\imath}} \bar{F}_{\bar{a}, i}-A_{\bar{\imath}}^{\bar{b}} \bar{F}_{\bar{b}, i}\right)\left(i \alpha_{-} \psi_{+}^{i}\right) \\
& =\psi_{+}^{\bar{\imath}} \lambda_{-}^{\bar{a}}\left[\bar{\partial}_{\bar{\imath}} \bar{F}_{\bar{a}, i}+A_{\bar{\imath} \bar{a}, i}^{\bar{b}} \bar{F}_{\bar{b}}-\partial_{i}\left(A_{\bar{\imath} \bar{a}}^{\bar{b}} \bar{F}_{\bar{b}}\right)\right]\left(i \alpha_{-} \psi_{+}^{i}\right) \\
& =\psi_{+}^{\bar{\imath}} \lambda_{-}^{\bar{a}}\left[\partial_{i}\left(\bar{\partial}_{\bar{\imath}} \bar{F}_{\bar{a}}-A_{\bar{\imath} \bar{a}}^{\bar{b}} \bar{F}_{\bar{b}}\right)+A_{\bar{\imath} \bar{a}, i}^{\bar{b}} \bar{F}_{\bar{b}}\right]\left(i \alpha_{-} \psi_{+}^{i}\right) \\
& =\psi_{+}^{\bar{\imath}} \lambda_{-}^{\bar{a}}\left(\partial_{i} \bar{D}_{\bar{\imath}} \bar{F}_{\bar{a}}+F_{i \bar{\imath} a \bar{a}} h^{a \bar{b}} \bar{F}_{\bar{b}}\right)\left(i \alpha_{-} \psi_{+}^{i}\right) \text {, }
\end{aligned}
$$


where we have used $A_{\bar{\imath}}^{\bar{b}, i}=h^{a \bar{b}} F_{i \bar{\imath} a \bar{a}}$ in the last step. It follows that, in addition to requiring (4.1), supersymmetry imposes the constraint

$$
\partial_{i} \bar{D}_{\bar{\imath}} \bar{F}_{\bar{a}}+F_{i \bar{\imath} a \bar{a}} h^{a \bar{b}} \bar{F}_{\bar{b}}=0 .
$$

Various special cases of (4.3) were used in [7] to establish properties of Mathai-Quillen form analogues which arise in the corresponding heterotic Landau-Ginzburg models. In that paper, it was claimed that supersymmetry imposes those constraints. In this paper, we have worked out the details supporting that claim.

Open Access. This article is distributed under the terms of the Creative Commons Attribution License (CC-BY 4.0), which permits any use, distribution and reproduction in any medium, provided the original author(s) and source are credited.

\section{References}

[1] E. Witten, Phases of $N=2$ theories in two-dimensions, Nucl. Phys. B 403 (1993) 159 [hep-th/9301042] [INSPIRE].

[2] J. Distler and S. Kachru, (0,2) Landau-Ginzburg theory, Nucl. Phys. B 413 (1994) 213 [hep-th/9309110] [INSPIRE].

[3] A. Adams, A. Basu and S. Sethi, (0,2) duality, Adv. Theor. Math. Phys. 7 (2003) 865 [hep-th/0309226] [INSPIRE].

[4] I.V. Melnikov and S. Sethi, Half-Twisted (0,2) Landau-Ginzburg Models, JHEP 03 (2008) 040 [arXiv: 0712.1058] [INSPIRE].

[5] J. Guffin and E. Sharpe, A-twisted heterotic Landau-Ginzburg models, J. Geom. Phys. 59 (2009) 1581 [arXiv:0801.3955] [INSPIRE].

[6] I. Melnikov, S. Sethi and E. Sharpe, Recent Developments in $(0,2)$ Mirror Symmetry, SIGMA 8 (2012) 068 [arXiv:1209.1134] [INSPIRE].

[7] R.S. Garavuso and E. Sharpe, Analogues of Mathai-Quillen forms in sheaf cohomology and applications to topological field theory, J. Geom. Phys. 92 (2015) 1 [arXiv:1310.5754] [INSPIRE].

[8] J. Guffin and E. Sharpe, A-twisted Landau-Ginzburg models, J. Geom. Phys. 59 (2009) 1547 [arXiv:0801.3836] [INSPIRE]. 